\title{
Injury To Inferior Vena Cava, CTCAE
}

National Cancer Institute

\section{Source}

National Cancer Institute. Injury To Inferior Vena Cava, CT CAE. NCI Thesaurus. Code C56735.

A finding of damage to the inferior vena cava. 